\title{
Inhibition of hydroperoxy-, keto- and hydroxy-FAME by alpha- and delta-tocopherol at Rancimat conditions
}

Susana Marmesat ${ }^{1}$, Arturo Morales ${ }^{1}$, M. Victoria Ruiz-Méndez ${ }^{1}$, Gloria Márquez-Ruiz ${ }^{2}$ and Joaquín Velasco ${ }^{1, *}$

${ }^{1}$ Instituto de la Grasa, Consejo Superior de Investigaciones Cientificas (CSIC),

Campus Universidad Pablo de Olavide E46, Ctra. de Utrera km 1, E-41013 Sevilla, Spain

${ }^{2}$ Instituto de Ciencia y Tecnología de Alimentos y Nutrición, Consejo Superior de Investigaciones Cientificas (CSIC), c/ José Antonio Novais, 10, E-28040 Madrid, Spain

*To whom correspondence should be addressed:

Telephone: $\quad+34954611550$

Fax: $\quad+34954616790$

E-mail: jvelasco@ig.csic.es 


\section{Abstract}

2 The effects of $\alpha$ - and $\delta$-tocopherol on inhibition of hydroperoxides, keto and hydroxy 3 compounds at Rancimat conditions, i.e. $100{ }^{\circ} \mathrm{C}$ and air bubbling, were studied in

4 samples of fatty acid methyl esters (FAME) obtained from high linoleic (HL) and high 5 oleic $(\mathrm{HO})$ sunflower oils. Primary hydroperoxides from methyl linoleate and methyl 6 oleate and secondary keto and hydroxy compounds derived from methyl linoleate 7 hydroperoxides were analyzed by HPLC-UV-ELS. Different tocopherol concentrations, 8 namely, 10, 50, 100, 500 and $1000 \mathrm{mg} / \mathrm{kg}$, were tested. Irrespective of the lipid substrate 9 and the initial concentration of tocopherol, results showed that the content of 10 hydroperoxides accumulated during the induction period was remarkably higher in the 11 samples containing $\delta$-tocopherol. The relative concentrations of oleate hydroperoxides 12 in the HO samples were also higher in the presence of $\delta$-tocopherol. $\alpha$-Tocopherol was 13 more effective in inhibiting hydroperoxides at low levels, with $100 \mathrm{mg} / \mathrm{kg}$ as optimal 14 concentration, while $\delta$-tocopherol displayed optimal protection at $1000 \mathrm{mg} / \mathrm{kg}$. Under 15 the oxidation conditions applied, neither $\alpha$ - nor $\delta$-tocopherol showed a protective effect on hydroperoxide decomposition at any level assayed. Formation of keto- and hydroxy-

17 dienes was more related to the concentration of their hydroperoxide precursors.

18 Furthermore, both tocopherols gave rise to increased concentrations of ketodienes at 19500 and $1000 \mathrm{mg} / \mathrm{kg}$ compared to the controls. Such an effect was more pronounced for $20 \quad \alpha$-tocopherol and in the HL samples.

21 Key-words: Tocopherol, hydroperoxides, ketodienes, hydroxydienes, Rancimat. 
24 The antioxidant activity of different types of tocopherols has been intensively investigated in a variety of lipid substrates and at different oxidation conditions, as compiled in different reviews [1-3]. The antioxidant properties of tocopherols are

27 mainly due to their ability to donate their phenolic hydrogen to peroxyl radicals. 28 According to their chemical structures, the hydrogen-donating power decreases in the 29 order $\alpha>\beta>\gamma>\delta$. It is generally accepted that the relative antioxidant activity of the 30 tocopherols in vivo is in the order $\alpha>\beta>\gamma>\delta$; however, there is widespread confusion 31 regarding their relative activities in fats and oils [1]. While the relative order of 32 antioxidant activity found in an organic solvent agreed with their hydrogen-donating 33 power [4], a reversed order, i.e. $\delta>\gamma \approx \beta>\alpha$, was obtained in fats, oils and lipoproteins 34 in vitro [1]. Although this is not yet clearly understood, it is known that the activities of 35 tocopherols not only depend on their reactivities towards peroxyl radicals, but also on 36 their participation in side reactions that normally reduce and counteract their principal 37 action mode. These side reactions are dramatically affected by tocopherol concentrations, the type of lipid substrate and other factors [1].

In most of the studies addressed to evaluate the antioxidant activities of tocopherols, the peroxide value is normally applied because it provides information on the total 41 hydroperoxides, i.e. the most abundant oxidation products formed at ambient 42 conditions. However, few are the studies in which the inhibitory effects of different 43 types of tocopherols on specific oxidation compounds have been undertaken.

44 The action of tocopherols goes further beyond their reduction effect on lipid oxidation rate. Tocopherols can also affect the relative abundance of lipid oxidation products

46 because of kinetic reasons. The isomerization of the cis, trans forms of methyl linoleate 
47 hydroperoxides to trans, trans isomers has been reported to be completely inhibited in the presence of high levels of $\alpha$-tocopherol [5-7]. However, hydroperoxides isomerized into trans,trans isomers at low $\alpha$-tocopherol concentrations $[6,7] \cdot \gamma$ - and $\delta$-Tocopherols also showed the same behavior, although their effects were quite lower than that of $\alpha$ tocopherol, resulting in a different relative isomeric distribution of hydroperoxides [6, 7].

Tocopherols have also been reported to inhibit hydroperoxide decomposition and affect the composition of derived products. Frankel and Gardner [8] found that the total amount of volatile compounds formed by thermal decomposition from a mixture of methyl linoleate hydroperoxides decreased in the presence of $\alpha$-tocopherol and that the relative distribution of individual volatiles also was affected. Koskas et al. [6] found that $\alpha-, \gamma-$ and $\delta$-tocopherols inhibited the decomposition of both 13-cis,trans and 13trans,trans linoleic acid hydroperoxides in water and that the activity order of 60 tocopherols was $\alpha-<\gamma-<\delta$-. Mäkinen et al. [7] also observed that $\gamma$ - and $\delta$-tocopherols were, in contrast to hydroperoxide isomerization, more efficient inhibitors of the decomposition of methyl linoleate hydroperoxides than $\alpha$-tocopherol. In addition, Huang et al. $[9,10]$ observed that $\alpha-, \gamma$ - and $\delta$-tocopherols inhibited formation of hexanal, a volatile compound formed from hydroperoxides of n-6 polyunsaturated fatty acids, and that, in contrast to hydroperoxide formation, the inhibition of hexanal 66 increased at higher levels of tocopherols. Nevertheless, Mäkinen et al. [7] observed a

67 loss of antioxidant efficiency of tocopherol in inhibiting hydroperoxide decomposition at high antioxidant concentration, being higher for $\alpha$-, but it was also evident for $\gamma$ - and

$69 \delta$-tocopherols. These authors suggested that the differences in the relative effects of tocopherols at different concentrations seem to result from a compromise between their 
radical scavenging efficiency and their participation in side reactions of peroxidizing nature.

In a previous study carried out in our lab [11], it was found that addition of $\alpha$ tocopherol in a FAME sample derived from high oleic sunflower oil resulted in changes in the distribution of hydroperoxides from methyl linoleate and methyl oleate. For a given content of total hydroperoxides, determined by the peroxide value, it was found that the relative concentration of methyl linoleate hydroperoxides increased in the presence of $\alpha$-tocopherol, whereas that of methyl oleate hydroperoxides decreased.

Quantitative analysis of methyl linoleate hydroperoxides was performed by an HPLC-

80 UV method developed in our lab [12]. The method allows the quantitative analysis of 81 the main oxidation products of linoleic acid, i.e. hydroperoxy-, keto- and hydroxy82 dienes, and it can be directly applied to samples of FAME obtained from vegetable oils. 83 Then the method was adapted to the analysis of vegetable oils by applying an adequate 84 derivatization step to transform the oxidized triacylglycerol molecules into FAME [14]. 85 In addition, the incorporation in series of an Evaporative Light Scattering detector 86 (ELS) proved to be useful for the analysis of methyl oleate hydroperoxides [14, 15].

87 Compared to a control, increased formation of keto- and hydroxy-dienes, both derived 88 from methyl linoleate hydroperoxides, was also found in the presence of $\alpha$-tocopherol 89 [11]. It was suggested that such an effect of $\alpha$-tocopherol could be related to its 90 participation in side reactions, through direct reactions with hydroperoxides to produce 91 secondary compounds.

92 The results of our previous study led us to go into detail on the inhibitory effects of 93 tocopherol through new kinetic studies. The present work was then undertaken to 94 examine the effect of the type of tocopherol, namely, $\alpha$ - and $\delta$-tocopherol, on the 
95 inhibition of lipid oxidation products. These tocopherols were chosen because they are

96 the two that differ most in their hydrogen-donating abilities [1]. FAME obtained from

97 high oleic (HO) and high linoleic (HL) sunflower oils were used as lipid substrates.

98 Unlike our previous study, in which a UV detector was used only, quantitative analysis

99 of hydroperoxides, keto and hydroxy compounds was performed by HPLC-UV-ELS

100 [14]. Different concentrations of both tocopherols, namely, 10, 50, 100, 500 and 1000

$101 \mathrm{mg} / \mathrm{kg}$, were assayed to study the influence of their hydrogen donating power on the

102 inhibition of compounds. For practical purposes, the oxidation conditions used were

103 those normally applied in the Rancimat test, i.e. $100{ }^{\circ} \mathrm{C}$ and air bubbling, which is an

104 accelerated method used to evaluate oxidative stability of oils.

105 
107 Chemicals

$108( \pm)$ - $\alpha$-Tocopherol $(>96 \%)$ and $(+)-\delta$-Tocopherol $(>90 \%)$ were purchased from Sigma109 Aldrich ${ }^{\circledR}$ (St. Louis, MO). Diethyl ether stabilized with 1\% v/v ethanol (Super purity 110 solvent, HPLC grade) was purchased from Romil, LTD (Cambridge, UK) and n111 heptane (99\% purity, HPLC grade) from Carlo Erba Reactifs-SDS (Val de Reuil,

112 France). Both HPLC solvents were used as received. All other chemicals and reagents

113 were of analytical grade and obtained from local suppliers.

114 Samples

115 Refined HL and HO sunflower oils were acquired in a local supermarket. Their fatty 116 acid compositions were $6.5 \% \mathrm{C} 16: 0,0.2 \% \mathrm{C} 16: 1,3.2 \% \mathrm{C} 18: 0,36.0 \% \mathrm{C} 18: 1,52.9 \%$

$117 \mathrm{C} 18: 2$ and 1.2\% others for HL, and 4.5\% C16:0, 0.2\% C16:1, 2.6\% C18:0, 81.3\%

$118 \mathrm{C} 18: 1,9.9 \% \mathrm{C} 18: 2$ and 1.5\% others for HO sunflower oil. Samples of FAME were

119 obtained from these two oils by transmethylation at room temperature with $\mathrm{KOH}$ in 120 methanol. Sixty grams of oil were dissolved in $200 \mathrm{~mL}$-hexane and $20 \mathrm{~mL}$ of a 121 solution $2 \mathrm{M} \mathrm{KOH}$ in methanol was added. Stirring was applied for $30 \mathrm{~min}$ and then the 122 medium was neutralized with $50 \mathrm{~mL}$ of $1 \mathrm{M} \mathrm{H}_{2} \mathrm{SO}_{4}$ in methanol. The organic phase was 123 then separated, washed with $80 \mathrm{~mL}$ water and filtered through filter paper containing 124 anhydrous $\mathrm{Na}_{2} \mathrm{SO}_{4}$. The solvent was evaporated in a rotary evaporator at $40{ }^{\circ} \mathrm{C}$. Finally, $125 \mathrm{~N}_{2}$ was bubbled through the lipid sample to remove the residual solvent. Thin layer 126 chromatography (TLC) was used to confirm that the transmethylation reaction was 127 complete. Silica plates and hexane:diethyl ether:acetic acid (80:20:1, v/v/v) as the 128 mobile phase were used. The spots on the plate were revealed with iodine vapor. The 129 FAME were purified by alumina column chromatography according to Yoshida et al. 
130 [16] to obtain FAME devoid of their naturally occurring tocopherols, peroxides and

131 trace metals. The samples obtained showed similar fatty acid compositions to their 132 parent oils.

133 When indicated, $\alpha$ - and $\delta$-tocopherol were added to the stripped FAME samples. An 134 aliquot of neat $\alpha$ - or $\delta$-tocopherol was weighed into a flat-bottomed round flask and 135 then the FAME sample was added to obtain a tocopherol concentration of $1000 \mathrm{mg} / \mathrm{kg}$ 136 sample. Stirring was applied for $15 \mathrm{~min}$ at room temperature. Then, five additional 137 samples containing $500,100,50$ and $10 \mathrm{mg} / \mathrm{kg}$ of $\alpha$ - or $\delta$-tocopherol were obtained by 138 dilution using purified FAME.

\section{Oxidation conditions}

140 Lots of six samples of $8 \pm 0.01 \mathrm{~g}$ containing $0,10,50,100,500$ and $1000 \mathrm{mg} / \mathrm{kg}$ of $\alpha-$

141 or $\delta$-tocopherol were placed into Rancimat tubes and these were inserted into the

142 heating block of a Rancimat device (Metrohm, Herisau, Switzerland), previously heated

143 at $100{ }^{\circ} \mathrm{C}$. The range of oxidation time studied was chosen from previous Oil Stability

144 Index (OSI) data provided by the Rancimat test at $100^{\circ} \mathrm{C}$. The air flow was $20 \mathrm{~L} \mathrm{~h}^{-1}$ in 145 all experiments. Samplings of $0.5 \mathrm{~g}$ were carried out along the induction period and the 146 samples were kept at $-35^{\circ} \mathrm{C}$ until analyses.

147 Analysis of oxidation compounds by HPLC

148 The main oxidation products of methyl linoleate and methyl oleate, namely, 149 hydroperoxides, keto- and hydroxy- compounds, were determined quantitatively by an 150 HPLC-UV-ELS method developed in our lab $[12,14]$. The oxidized FAME samples 151 were analyzed in a Waters 600 HPLC chromatograph. The chromatograph was 152 equipped with a Waters 600 pump, a Rheodyne injector valve (20- $\mu \mathrm{L}$ sample loop), a 
153 silica HPLC column (LiChrospher ${ }^{\circledR} \mathrm{Si} 60,250 \mathrm{~mm}$ x $4 \mathrm{~mm}$ i.d., $5 \mu \mathrm{m}$ particle size)

154 (Merck, Darmstadt, Germany), an HP 1050 Series variable wavelength UV detector (8 155 mm path length) (Agilent Technologies Inc., Palo Alto, CA), a Waters 2424 156 Evaporative Light Scattering (ELS) detector, an Agilent 35900E A/D Converter 157 (Agilent Technologies Inc.) and a Waters 600 controller. The separation of analytes was 158 performed in isocratic elution using n-heptane:diethyl ether $(82: 18, \mathrm{v} / \mathrm{v})$ with a flow rate 159 of $1 \mathrm{~mL} / \mathrm{min}$. Ethanol present in diethyl ether as a stabilizer was not removed. 160 Hydroperoxy- and hydroxy-dienes were recorded by the UV detector at $234 \mathrm{~nm}$, while 161 ketodienes were at $268 \mathrm{~nm}$. Hydroperoxides from methyl oleate and methyl linoleate as 162 a whole were detected by the ELS. The ELS conditions applied were $35^{\circ} \mathrm{C}$ for nebulizer 163 temperature, $40{ }^{\circ} \mathrm{C}$ for tube temperature and 25 psi for gas flow. The gas used for 164 nebulization was nitrogen (purity 99.99\%). The gain was set at 50. Quantitative data by 165 the UV detector were obtained by applying response factors reported elsewhere [12], 166 while external calibration was applied for quantitative determination of compounds by 167 the ELS, according to a previous report [14].

168 Analysis of tocopherols

169 Analysis of $\alpha$ - and $\delta$-tocopherol was carried out by normal-phase HPLC with 170 fluorescence detection following IUPAC standard method 2.411 [17].

171 Oil stability index (OSI)

172 The OSI of the FAME samples was determined at $100{ }^{\circ} \mathrm{C}$ according to AOCS Official 173 Method Cd 12b-92 [18]. A Rancimat instrument, model 679, from Metrohm (Metrohm,

174 Herisau, Switzerland) was used in this study. The tests were carried out with $2.5 \mathrm{~g}$ of oil 175 and with an air flow of $20 \mathrm{~L} \mathrm{~h}^{-1}$. 
176 Statistical analysis

177 Experiments were run in triplicate and results were presented as mean values of three

178 analytical determinations. Comparisons were made by the Student's $t$ test using

179 Microsoft Excel 2010 (Microsoft Corporation, Redmond, WA, USA). Linear regression

180 analysis was also performed in Microsoft Excel 2010. Significance was defined at $p<$

$181 \quad 0.05$.

182 


\section{Inhibition of hydroperoxides}

185 Formation of hydroperoxides in the two lipid substrates, HL and HO, followed different

186 reaction kinetics in the presence of $\alpha$ - and $\delta$-tocopherol. In the samples containing $\alpha$ -

187 tocopherol, the formation of hydroperoxides took place at constant rate during the

188 whole induction period (IP), i.e. until the total loss of the antioxidant occurred (Fig. 1).

189 As a result, the reaction order for hydroperoxides in both lipid substrates was zero in the

190 presence of $\alpha$-tocopherol at all the concentrations assayed. On the other hand,

191 hydroperoxides only increased linearly during the first stages of oxidation in the

192 samples containing $\delta$-tocopherol and the reaction rate increased progressively in the

193 presence of substantial contents of the antioxidant. This was a common pattern for all

194 the samples analyzed, i.e. irrespective of the lipid substrate and the initial concentration

195 of $\delta$-tocopherol (Fig. 1). Compared to the samples containing $\alpha$-tocopherol, the content

196 of hydroperoxides accumulated during the IP was remarkably higher in the presence of

$197 \delta$-tocopherol at any initial tocopherol concentration assayed and in both lipid substrates.

198 Given that hydroperoxides are the major oxidation compounds formed during the IP and

199 that their participation in other reactions is relatively low compared to their formation, a

200 rough estimate of the oxidation rate can be expressed as Eq. 1 [19].

$$
\frac{\mathrm{d}[\mathrm{LOOH}]}{\mathrm{dt}}=\mathrm{k} \cdot[\mathrm{LOOH}]^{\mathrm{n}}
$$

202 The kinetic parameters $k$ and $n$ represent a global oxidation rate constant and the reaction order, respectively. By rearrangement and integration of Eq. 1, Eq. 2 is readily obtained, in which $[\mathrm{LOOH}]$ and $[\mathrm{LOOH}]_{0}$ represent the concentrations of hydroperoxides at time $t$ and 0 , respectively. 


$$
[\mathrm{LOOH}]^{1-\mathrm{n}}=[\mathrm{LOOH}]_{0}^{1-\mathrm{n}}+\mathrm{kt}
$$

207 The kinetic parameters $k$ and $n$ were calculated from the experimental data acquired 208 during the whole IP. The results obtained showed that all the samples containing $\delta$ 209 tocopherol presented similar reaction kinetics to the control samples, i.e. in the absence 210 of antioxidant, in terms of the reaction order, which was equal to 0.5 (Table 1).

211 The different oxidation kinetics displayed by the samples containing different type of 212 tocopherol along with the kinetic similarities between the samples containing $\delta$ 213 tocopherol and the control samples can be attributed to the different hydrogen donating 214 abilities of the two antioxidants, being much larger for $\alpha$-tocopherol [1]. Compared to $215 \delta$-, the larger reactivity of $\alpha$-tocopherol towards peroxyl radicals gave rise to a more 216 effective inhibition of hydroperoxide formation during the whole IP since 217 hydroperoxides increased at constant rate until the antioxidant was completely depleted.

218 The fact that the oxidation rate did not increase until the total loss of $\alpha$-tocopherol took

219 place is consistent with the fact that such a kinetic pattern was even observed when the 220 lowest antioxidant concentration was assayed $(10 \mathrm{mg} / \mathrm{kg})$, emphasizing the enormous 221 hydrogen donating power of $\alpha$-tocopherol and its effectiveness at low concentrations.

222 On the other hand, $\delta$-tocopherol was not reactive enough and the rate of hydroperoxide 223 formation increased in the presence of still considerably high contents of the 224 antioxidant. In consequence, larger contents of hydroperoxides were accumulated 225 during the IP in the presence of $\delta$-tocopherol compared to $\alpha$-. Accumulation of 226 oxidation compounds during the IP of lipid model systems and vegetable oils has been 227 reported to decrease when temperature was increased $[20,21]$ and to increase as the 228 degree of unsaturation of the oil was higher [22]. However, to the best of our 229 knowledge, the results of the present study are the first evidence of that the type of 
230 tocopherol can also have a remarkable impact on the total amounts of oxidation

231 products accumulated during the IP.

232 The differences in the hydrogen donating capabilities between $\alpha$ - and $\delta$-tocopherol also

233 gave rise to differences in the relative formation of hydroperoxides from methyl oleate

234 and methyl linoleate in the HO samples. As to the HL samples, the oxidation of methyl

235 oleate was considerably low (not shown), which supports previous results [11]. The

236 concentration of the primary hydroperoxides of methyl oleate can be determined by

237 difference from the results obtained with the two detectors used in series, UV and ELS

238 [14]. In a previous report it was found that substantial contents of methyl oleate

239 hydroperoxides were detected in FAME from HO sunflower oil in the absence of

240 antioxidant [11]. When $\alpha$-tocopherol was added the oxidation of methyl oleate was

241 remarkably reduced compared to methyl linoleate. In the present study, $\alpha$-tocopherol

242 considerably inhibited the formation of methyl oleate hydroperoxides in the HO sample

243 at all the concentrations assayed, whereas substantial oxidation of methyl oleate was

244 detected in the presence of $\delta$-tocopherol. In this regard, the relationship between the

245 contents of hydroperoxides detected by ELS and hydroperoxydienes by UV was linear

246 during the entire IP in all the HO samples containing $\alpha$-tocopherol. No differences were

247 found in such a relationship between the samples containing different initial

248 concentrations of $\alpha$-tocopherol (Fig. 2a). The oleate hydroperoxides did not constitute

249 more than $19 \mathrm{~mol} \%$ of the analyzed hydroperoxides at the end of the IP in all the

250 samples containing $\alpha$-tocopherol. In contrast, the formation of hydroperoxides from

251 methyl oleate seemed to be dependent of the initial concentration of $\delta$-tocopherol (Fig.

252 2b). For a given content of total hydroperoxides formed during the IP, the relative

253 amount of oleate hydroperoxides was lower when the initial concentration of $\delta$ - 
tocopherol was increased. In addition, unlike the samples containing $\alpha$-tocopherol, the relative concentration of oleate hydroperoxides formed during the IP increased with the oxidation extent in the presence of $\delta$-tocopherol. In fact, the relationship between total hydroperoxides and hydroperoxydienes was not linear (Fig. 2b). The data adjusted well to quadratic polynomial functions (not shown). The oleate hydroperoxides levels at the end of the IP were approximately $50 \mathrm{~mol} \%$ of the analyzed hydroperoxides in all the samples containing $\delta$-tocopherol.

261 It has been reported that $\alpha$-tocopherol was more efficient in inhibiting the isomerization of cis,trans methyl linoleate hydroperoxides to the trans,trans isomers than $\delta$ -

263 tocopherol due to its higher hydrogen donating ability [7]. Nevertheless, no reported 264 data have been found in relation to the relative hydroperoxide formation of methyl 265 oleate and methyl linoleate. The analytical method used in the present study has allowed us to determine how the type of tocopherol can influence the relative formation of hydroperoxides of different unsaturated fatty acids. In the present study, and also in a

268 previous report [11], it was found that $\alpha$-tocopherol considerably reduced the oxidation 269 of methyl oleate in a HO sample compared to a control, i.e. in the absence of 270 antioxidant. Due to its great reactivity with peroxyl radicals, $\alpha$-tocopherol largely 271 prevented the participation of methyl oleate in the propagation step of the oxidation 272 process. The larger formation of methyl oleate hydroperoxides in the HO samples 273 containing $\delta$-tocopherol could be therefore explained by a slower interaction of the 274 antioxidant with peroxyl radicals, thus allowing peroxyl radicals to react with methyl 275 oleate in the propagation step to a greater extent.

276 Given the different kinetics shown by the samples containing each type of tocopherol, 277 the initial rate of inhibited oxidation $\left(\mathrm{W}_{\mathrm{inh}}\right)$ was taken into account to compare the 
278 effects of the two antioxidants [23]. The amounts of hydroperoxides found in the early 279 stages of oxidation for the controls and the samples containing $\delta$-tocopherol were 280 considered for calculation of pseudo zero-order rate constants. As to the samples 281 containing $\alpha$-tocopherol, all the data obtained during the IP were used in the analysis 282 and, in consequence, the initial rate constants were the same as the global rate constants 283 during the whole IP. As expected, the results showed that the $\mathrm{W}_{\text {inh }}$ decreased to a great 284 extent in the presence of either tocopherol (Table 2). The decrease in $\mathrm{W}_{\text {inh }}$ was in line 285 with the increase of antioxidant concentration up to 50 and $500 \mathrm{mg} / \mathrm{kg}$ for $\alpha$ - and $\delta$ 286 tocopherol, respectively, in the HL sample and up to $50 \mathrm{mg} / \mathrm{kg}$ for both tocopherols in 287 the HO sample. Then a significant gradual increase was observed with tocopherol 288 concentration. This significant increase in the oxidation rate was not observed in the rate 289 constants presented in Table 1. When the whole IP was considered in the samples 290 containing $\delta$-tocopherol, the oxidation rate decreased as the initial content of the 291 antioxidant was increased.

292 In both lipid substrates, the initial oxidation rate was lower in the samples containing $\alpha$ 293 tocopherol when the concentration was equal to or lower than $100 \mathrm{mg} / \mathrm{kg}$ (Table 2). No 294 significant differences between the two tocopherols were found at $500 \mathrm{mg} / \mathrm{kg}$ and $\delta$ 295 tocopherol was more effective in reducing the oxidation rate at $1000 \mathrm{mg} / \mathrm{kg}$ in the two 296 lipid substrates.

297 For a better characterization of the inhibitory effect of the two antioxidants on 298 hydroperoxides, the IP, which was determined as the time at which oxidation was 299 accelerated or when the total loss of tocopherol took place, was also evaluated. While 300 the $\mathrm{W}_{\text {inh }}$ reflects the antioxidant strength, the IP estimates the effectiveness of an 301 antioxidant in terms of its ability to terminate the chain radical process by reacting with 
302 the peroxyl radicals, which are responsible for the duration of the IP. The results

303 obtained showed differences in the effectiveness of the two tocopherols between the two

304 lipid substrates (Fig. 3a-b). No significant differences were found between the two

305 tocopherols at 10 and $500 \mathrm{mg} / \mathrm{kg}$ in the HL sample. However, $\alpha$ - was more effective

306 than $\delta$-tocopherol at 50 and $100 \mathrm{mg} / \mathrm{kg}$ and the opposite was found at $1000 \mathrm{mg} / \mathrm{kg}$. As to

307 the HO samples, no significant differences were found between the two antioxidants at

308 low concentrations, i.e. 10 and $50 \mathrm{mg} / \mathrm{kg}$, and $\delta$ - was significantly more effective than

$309 \alpha$-tocopherol from $100 \mathrm{mg} / \mathrm{kg}$. These results were consistent with the oxidative stability

310 index (OSI) values provided by the Rancimat test (Fig. 3a'-b').

311 The stabilization factor $(F)$, defined as the ratio between the IPs in the presence and

312 absence of antioxidant, increased linearly with the initial concentration of both

313 tocopherols up to $100 \mathrm{mg} / \mathrm{kg}$ in the two lipid substrates. Afterwards, deviation from

314 linearity took place (Fig. 4). It is known that the absence of linearity is due to the

315 participation of the antioxidant in side reactions, generating free radicals during the

316 propagation and the consequent loss of efficacy [23]. Therefore, both tocopherols

317 showed loss of effectiveness from concentrations larger than $100 \mathrm{mg} / \mathrm{kg}$, which is

318 consistent with the loss of efficacy in reduction the initial oxidation rate shown by the

319 two tocopherols. Yanishlieva et al. [24] suggested that both $\alpha$ - and $\gamma$-tocopherol lose

320 their efficacy at high concentrations owing to their direct reaction with hydroperoxides,

321 giving alkoxyl radicals that may amplify the rate of lipid oxidation by participating in

322 propagation reactions. In addition, it was also suggested that tocopheroxyl radicals can

323 also participate in a number of peroxidizing reactions propagating the oxidation chain.

324 These authors found that $\alpha$-tocopherol participated more readily in side reactions than $\gamma$ -

325 tocopherol, which was attributed to its higher hydrogen-donating power. 
326 In order to determine the most optimal concentrations of the two tocopherols, the

327 kinetic parameter $A$ proposed by Yanishlieva and Marinova [25] to evaluate antioxidant

328 activity was examined (Table 3 ). This parameter is calculated by the ratio between the

329 parameter $F$ and the oxidation rate ratio $(O R R)$, defined as the ratio between the initial

330 oxidation rates in the presence and absence of antioxidant. Results showed that $\alpha$ -

331 tocopherol was most effective at $100 \mathrm{mg} / \mathrm{kg}$, while $\delta$-tocopherol displayed optimal

332 protection at $1000 \mathrm{mg} / \mathrm{kg}$ in both lipid substrates. If data in Table 1 had been considered

333 for calculation of $A$ instead of the initial oxidation rates, the optimal concentration for $\delta$ -

334 tocopherol would have also been the highest concentration assayed, i.e. $1000 \mathrm{mg} / \mathrm{kg}$.

335 Although $\alpha$-tocopherol was obviously more effective in prolonging the IP at 1000

$336 \mathrm{mg} / \mathrm{kg}$ than at $100 \mathrm{mg} / \mathrm{kg}$, the opposite was found for the reduction of the initial

337 oxidation rate. Unlike the Rancimat test, which provides the time at which the

338 antioxidants are exhausted, the parameter $A$ also takes into account the ability of an

339 antioxidant to change the initial oxidation rate. From a practical point of view, this is

340 essential because the appearance of rancidity occurs at relatively low oxidation extents,

341 which are normally reached far earlier than when the antioxidants are completely

342 consumed.

343 Inhibition of keto- and hydroxy-dienes

344 Supporting previous studies [14], secondary oxidation products of methyl oleate

345 hydroperoxides were not detected by the ELS in any of the samples analyzed. In those

346 samples in which methyl oleate hydroperoxides were formed significantly, i.e. the

347 control HO sample and the HO samples containing $\delta$-tocopherol, the absence of such

348 compounds could be attributed to the low sensitivity of the ELS compared to the UV

349 detector. 
350 Keto- and hydroxy-dienes showed similar patterns to their precursors, i.e.

351 hydroperoxydienes, in the samples containing tocopherol. Slight formation of

352 ketodienes was observed and, as expected, a sharp increase took place when tocopherol

353 was completely depleted (not shown). On the other hand, hydroxydienes were not

354 detected in the presence of antioxidant in the samples containing 10,50 and $100 \mathrm{mg} / \mathrm{kg}$

355 of either $\alpha$ - or $\delta$-tocopherol, whereas in the samples containing 500 and $1000 \mathrm{mg} / \mathrm{kg}$

356 formation of the hydroxy compounds was observed when the content of remaining

357 tocopherol was low (not shown). Similarly, a sharp increase of hydroxydienes occurred

358 when tocopherol was exhausted.

359 As more hydroperoxides were formed during the IP in the samples containing $\delta$ -

360 tocopherol, more decomposition products were also found in these samples. However,

361 neither $\alpha$-nor $\delta$-tocopherol at levels lower than $500 \mathrm{mg} / \mathrm{kg}$ showed a protective effect

362 on the hydroperoxide decomposition. Thus, for a given content of hydroperoxydienes,

363 similar concentrations of ketodienes were found between the samples containing

364 tocopherol and the control samples (Fig. 5a-a').

365 When the higher concentrations of both tocopherols were assayed, i.e. 500 and 1000

$366 \mathrm{mg} / \mathrm{kg}$, it was observed that formation of ketodienes was favored with respect to their

367 precursors, i.e. hydroperoxydienes. In this regard, for a given concentration of

368 hydroperoxydienes, significantly larger contents of ketodienes were found in these

369 samples compared to the controls. This effect was more pronounced in the samples

370 containing $\alpha$-tocopherol and in the more unsaturated sample, i.e. the HL sample (Fig.

371 5). As to hydroxydienes, such an effect was not observed. In this regard, their contents

372 were similar to those found in the controls for a given content of hydroperoxydienes 373 (not shown). 
374 From the results obtained it can be said that under the oxidation conditions applied,

375 neither $\alpha$ - nor $\delta$-tocopherol showed a protective effect on hydroperoxide decomposition

376 at any level assayed. Formation of keto- and hydroxy-dienes was more related to the

377 concentration of their hydroperoxide precursors. However, at high tocopherol

378 concentrations, i.e. 500 and $1000 \mathrm{mg} / \mathrm{kg}$, both tocopherols gave rise to increased

379 concentrations of ketodienes compared to the control samples, whereas such an effect

380 was not observed for hydroxydienes. These results are in agreement with previous

381 studies [11] that showed increased formation of keto- and also, in a lower extent,

382 hydroxy-dienes in the presence of $500 \mathrm{mg} / \mathrm{kg} \alpha$-tocopherol compared to a control. It

383 was suggested that due to its high hydrogen-donating capability, $\alpha$-tocopherol could

384 react directly with hydroperoxides, yielding alkoxyl radicals, as commented above, and

385 so acting as a decomposer of hydroperoxides $[24,26]$. The larger effect found for $\alpha$ -

386 compared to $\delta$-tocopherol in this study can be attributed to its higher hydrogen-donating

387 power. These results are in agreement with those reported by Mäkinen et al. [7], who

388 observed a major decomposing effect of $\alpha$ - than $\delta$-tocopherol on pure linoleate

389 hydroperoxides. Similarly, the fact that the increased formation of ketodienes compared

390 to a control sample was only observed at high concentrations of tocopherols can also be

391 attributed to the increase of the hydrogen-donating activity with the antioxidant

392 concentration [7]. In addition, the fact that such an effect was more pronounced in the

393 HL than in the HO sample could be related to the much higher relative concentration of

394 the more reactive methyl linoleate hydroperoxides found in the HL sample. In this

395 respect, Yanishlieva et al. [24] found rate constants for the participation of $\alpha$ - and $\gamma$ -

396 tocopherol in one side reaction that were higher in purified soybean oil than in

397 sunflower oil. The authors suggested that these results may have been due to the higher

398 reactivity of the linolenate hydroperoxides that are formed in soybean oil. 
399 The fact that a protective role of tocopherols on the decomposition of hydroperoxides

400 was not observed in the present study is in contrast with the studies of Mäkinen et al. [7,

401 27]. This could be related to differences in the oxidation conditions applied.

402 Specifically, the oxidation temperature used in the present study $\left(100^{\circ} \mathrm{C}\right)$ was quite 403 different from that applied by Mäkinen and coworkers $\left(40^{\circ} \mathrm{C}\right)$. The isomerization of the

404 cis,trans methyl linoleate hydroperoxides to the trans,trans isomers has been reported to

405 precede the hydroperoxide decomposition and hence the protective effect of tocopherol

406 seems to be due to its capability to inhibit such an isomerization reaction [27]. In this

407 regard, the larger formation of trans, trans isomers of hydroperoxides with temperature

408 [28] could explain the lack of tocopherol activity in protecting hydroperoxides from 409 decomposition found in the present study.

\section{Conclusions}

411 The results of this study have shown that different relative concentrations of oxidation

412 products and different global amounts of compounds formed during the IP may result

413 from each type of tocopherol. The combination of the IPs with the initial oxidation rate

414 data to evaluate the efficacy of antioxidants under conditions similar to the Rancimat

415 test constitutes a more complete approach than the Rancimat test, i.e. the OSI values.

416 Unlike the Rancimat test, which evaluates the time at which antioxidants are completely

417 consumed, such an approach also takes into account the initial stages of lipid oxidation.

418 Rancidity of oils normally appears at very low oxidation extents, which are reached in

419 the presence of elevated contents of antioxidants. Obviously, the Rancimat test is a

420 convenient method that provides useful information on the oxidative stability of oils.

421 The stability order of a series of oils provided by the Rancimat should be similar to the

422 one that would be obtained at room temperature, provided that oxygen is not limiting. 
423 Nevertheless, the different influence of the type and concentration of tocopherol on

424 reducing the initial oxidation rate and prolonging the IP may result in changes in the 425 expected order of stability at room temperature predicted by Rancimat.

\section{Acknowledgments}

427 This work was funded by the Spanish Ministry of Economy and Competitiveness 428 through project AGL2013-45110-R and CSIC through a PIE project with reference 429 201270E134. The authors also thank the Spanish Ministry of Economy and 430 Competitiveness for supporting Susana Marmesat with a "Juan de la Cierva" contract 431 with reference JCI-2011-08874.

432 


\section{References}

4341 Kamal-Eldin A, Appelqvist L $\AA$ (1996) The chemistry and antioxidant properties of 435 tocopherols and tocotrienols. Lipids 31(7):671-701

4362 Kamal-Eldin A (2006) Effect of fatty acids and tocopherols on the oxidative 437 stability of vegetable oils. Eur J Lipid Sci Technol 108(12):1051-1061

4383 Seppanen CM, Song Q, Csallany AS (2010) The antioxidant functions of 439 tocopherol and tocotrienol homologues in oils, fats, and food systems. J Am Oil $440 \quad$ Chem Soc 87(5):469-481

4414 Burton GW, Ingold, KU (1981) Autoxidation of biological molecules. 1. 442 Antioxidant activity of vitamin E and related chain-breaking phenolic antioxidants 443 in vitro. J Am Chem Soc 103(21):6472-6477

4445 Peers KE, Coxon DT, Chan H W-S (1981) Autoxidation of methyl linolenate and 445 methyl linoleate: The effect of $\alpha$-tocopherol. J Sci Food Agric 32:898-904

4466 Koskas JP, Cillard J, Cillard P (1984) Autoxidation of linoleic acid and behavior of 447 its hydroperoxides with and without tocopherols. J Am Oil Chem Soc 61:1466-1469

4487 Mäkinen M, Kamal-Eldin A, Lampi A-M, Hopia A (2001) $\alpha-,-\gamma$, and $\delta$ 449 Tocopherols as inhibitors of isomerization and decomposition of cis,trans methyl $450 \quad$ linoleate hydroperoxides. Eur J Lipid Sci Technol 103:286-291

4518 Frankel EN, Gardner HW (1989) Effect of $\alpha$-tocopherol on the volatile thermal 452 decomposition products of methyl linoleate hydroperoxides. Lipids 24(7):603-608

4539 Huang SW, Frankel EN, German JB (1994) Antioxidant activity of $\alpha$-and $\gamma$ 454 tocopherols in bulk oils and in oil-in-water emulsions. J Agric Food Chem $455 \quad 42(10): 2108-2114$ 
10 Huang SW, Frankel EN, German JB (1995) Effects of individual tocopherols and

457 tocopherol mixtures on the oxidative stability of corn oil triglycerides. J Agric Food

$458 \quad$ Chem 43(9):2345-2350

45911 Morales A, Dobarganes C, Márquez-Ruiz G, Velasco J (2012) Formation of 460 hydroperoxy-, keto- and hydroxy-dienes in FAME from oils: influence of 461 temperature and addition of $\alpha$-tocopherol. J Am Oil Chem Soc 89:675-684

46212 Morales A, Dobarganes C, Márquez-Ruiz G, Velasco J (2010) Quantitation of 463 hydroperoxy-, keto- and hydroxy-dienes during oxidation of FAMEs from high464 linoleic and high-oleic sunflower oils. J Am Oil Chem Soc 87:1271-1279

46513 Morales A, Marmesat S, Dobarganes C, Márquez-Ruiz G, Velasco J (2012) 466 Quantitative analysis of hydroperoxy-, keto- and hydroxy-dienes in refined 467 vegetable oils. J Chromatogr A 1229:190-197

46814 Morales A, Marmesat S, Dobarganes C, Márquez-Ruiz G, Velasco J (2012) 469 Evaporative light scattering detector in normal-phase high-performance liquid 470 chromatography determination of FAME oxidation products. J Chromatogr A $471 \quad 1254: 62-70$

47215 Morales A, Marmesat S, Ruiz-Méndez MV, Márquez-Ruiz G, Velasco J (2014) 473 Formation of oxidation products in vegetable oils analysed as FAME derivatives by 474 HPLC-UV-ELSD. Food Res Int 62:1080-1086

47516 Yoshida H, Kondo I, Kajimoto G (1992) Participation of free fatty acids in the 476 oxidation of purified soybean oil during microwave heating. J Am Oil Chem Soc $477 \quad 11: 1136-1140$

47817 IUPAC (1992) Standard Methods for the Analysis of Oils, Fats and Derivatives, 7th 479 edn. International Union of Pure and Applied Chemistry, Blackwell Scientific, $480 \quad$ Oxford, UK 
18 AOCS (1998) Official Methods and Recommended Practices of the American Oil

482 Chemists' Society, 5th edn., edited by D Firestone, AOCS Press, Champaign, $483 \quad$ Illinois.

48419 Márquez-Ruiz G, Martín-Polvillo M, Dobarganes C (2003) Effect of temperature 485 and addition of $\alpha$-tocopherol on the oxidation of trilinolein model systems. Lipids $486 \quad 38(3): 233-240$

48720 Márquez-Ruiz G, Martín-Polvillo M, Dobarganes C (1996) Quantitation of oxidized 488 triglyceride monomers and dimers as an useful measurement for early and advanced 489 stages of oxidation. Grasas y Aceites, 47(1-2):48-53

49021 Márquez-Ruiz G, Martín-Polvillo M, Velasco J, Dobarganes C (2008) Formation of 491 oxidation compounds in sunflower and olive oils under oxidative stability index conditions. Eur J Lipid Sci Technol 110(5):465-471

22 Martin-Polvillo M, Marquez-Ruiz G, Dobarganes MC (2004) Oxidative stability of 494 sunflower oils differing in unsaturation degree during long-term storage at room temperature. J Am Oil Chem Soc 81(6):577-583

49623 Yanishlieva NV, Marinova EM (2003) Kinetic evaluation of the antioxidant activity in lipid oxidation. In: Kamal-Eldin A (ed) Lipid oxidation pathways. AOCS Press, Champaign, pp 85-110

24 Yanishlieva NV, Kamal-Eldin A, Marinova EM, Toneva AG (2002) Kinetics of $500 \quad$ antioxidant action $\alpha$ - and $\gamma$-tocopherols in sunflower and soybean triacylglycerols. $501 \quad$ Eur J Lipid Sci Technol 104:262-270

50225 Yanishlieva NV, Marinova EM (1992) Inhibited oxidation of lipids. I: Complex 503 estimation of the antioxidative properties of natural and synthetic antioxidants. Fat $504 \quad$ Sci Technol 94: 374-379 
50526 Kamal-Eldin A, Mäkinen M, Lampi A-M, Hopia A (2002) A multivariate study of

$506 \alpha$-tocopherol and hydroperoxide interaction during the oxidation of methyl

$507 \quad$ linoleate. Eur Food Res Technol 214:52-57

50827 Mäkinen M, Hopia A (2000) Effects of $\alpha$-tocopherol and ascorbyl palmitate on the

509 isomerization and decomposition of methyl linoleate hydroperoxides. Lipids $510 \quad 35: 1215-1223$

51128 Frankel EN (2005) Hydroperoxide formation. In: Frankel EN (ed) Lipid oxidation.

512 The Oily Press, Bridgwater (UK), pp 25-50

513 


\section{$514 \quad$ Figure legends}

515 Fig. 1. Oxidative patterns found for FAME containing $\alpha$ - or $\delta$-tocopherol at $100{ }^{\circ} \mathrm{C}$. (a-

516 a') HL samples containing 100 and $1000 \mathrm{mg} / \mathrm{kg}$ tocopherol. (b-b') HO samples

517 containing 100 and $1000 \mathrm{mg} / \mathrm{kg}$ tocopherol. (Circles) Samples containing $\alpha$-tocopherol.

518 (Squares) Samples containing $\delta$-tocopherol. Solid symbols correspond to

519 hydroperoxides and hollow symbols to tocopherol.

520 Fig. 2. Relationships between hydroperoxides detected by ELS and hydroperoxydienes

521 by UV in the HO samples. (a) Samples containing $\alpha$-tocopherol. (b) Samples

522 containing $\delta$-tocopherol. The linear equation found for the data in $\mathbf{A}$ was $\mathrm{y}=1.21 \mathrm{x}$ with a

523 correlation coefficient of 0.9984.

524 Fig. 3. Induction periods and the oxidative stability index (OSI) values determined in

525 Rancimat. (a-a') HL samples. (b-b') HO samples. The induction periods for the HL and 526 HO control samples were 0.7 and $1.7 \mathrm{~h}$, respectively. The OSI values for the HL and 527 HO control samples were 2.23 and 5.12 h, respectively. Error bars express standard 528 deviation and asterisks denote significant differences $(p<0.05)$.

529 Fig. 4. Influence of tocopherol concentration on the stabilization factor $(F)$. (a) HL 530 samples. (b) $\mathrm{HO}$ samples. (Circles) Samples containing $\alpha$-tocopherol. (Squares) 531 Samples containing $\delta$-tocopherol. Data obtained from the OSI values.

532 Fig. 5. Relationships between keto- and hydroperoxy-dienes in the HL (a-c) and HO 533 (a'-c') samples at different concentrations of tocopherols: $100 \mathrm{mg} / \mathrm{kg}(\mathbf{a}-\mathbf{a}$ '), $500 \mathrm{mg} / \mathrm{kg}$ 534 (b-b') and $1000 \mathrm{mg} / \mathrm{kg}$ (c-c'). (Triangles) Control samples, (squares) samples 535 containing $\alpha$-tocopherol, (circles) samples containing $\delta$-tocopherol. 
Table 1 Global rate constants $\left(k(\mathrm{mmol} / \mathrm{kg} \mathrm{s})^{0.5}\right.$ x $\left.10^{4}\right)$ and linear correlation coefficients $(r)$ for hydroperoxide formation during the induction period of samples containing $\delta$ tocopherol considering a reaction order of 0.5 .

\begin{tabular}{lccccc}
\hline \multirow{2}{*}{ Toc $(\mathbf{m g} / \mathbf{k g})$} & \multicolumn{2}{c}{ HL } & & \multicolumn{2}{c}{ HO } \\
\cline { 2 - 3 } \cline { 5 - 6 } \cline { 5 - 6 } & $13.8 \pm 0.4 \mathrm{a}$ & 0.9986 & & $5.0 \pm 0.3 \mathrm{a}$ & 0.9957 \\
\hline 0 & $5.2 \pm 0.4 \mathrm{~b}$ & 0.9974 & & $1.10 \pm 0.09 \mathrm{~b}$ & 0.9899 \\
10 & $2.6 \pm 0.2 \mathrm{c}$ & 0.9913 & & $0.54 \pm 0.02 \mathrm{c}$ & 0.9968 \\
50 & $1.9 \pm 0.1 \mathrm{~d}$ & 0.9949 & & $0.54 \pm 0.02 \mathrm{c}$ & 0.9978 \\
100 & $1.31 \pm 0.09 \mathrm{e}$ & 0.9917 & & $0.32 \pm 0.01 \mathrm{~d}$ & 0.9959 \\
500 & $1.13 \pm 0.05 \mathrm{f}$ & 0.9941 & & $0.33 \pm 0.01 \mathrm{~d}$ & 0.9942 \\
1000 & & & & \\
\hline
\end{tabular}

Different letters for different treatments in each lipid sample denote significant differences $(p<0.05)$. 
Table 2 Initial oxidation rates (W (mmol/ $\mathrm{kg} \mathrm{s})$ x $10^{4}$ ) for hydroperoxide formation.

\begin{tabular}{lcccc}
\hline Toc $(\mathbf{m g} / \mathbf{k g})$ & HL- $\alpha$ & HL- $\delta$ & HO- $\alpha$ & HO- $\delta$ \\
\hline 0 & $25.6 \pm 3.1 \mathrm{~d}$ & $25.6 \pm 3.1 \mathrm{e}$ & $3.5 \pm 0.9 \mathrm{f}$ & $3.5 \pm 0.9 \mathrm{e}$ \\
10 & $4.0 \pm 0.4 \mathrm{bA}$ & $7.9 \pm 0.9 \mathrm{~dB}$ & $0.40 \pm 0.02 \mathrm{bA}$ & $1.17 \pm 0.13 \mathrm{cB}$ \\
50 & $2.0 \pm 0.1 \mathrm{aA}$ & $5.8 \pm 0.2 \mathrm{cB}$ & $0.36 \pm 0.02 \mathrm{aA}$ & $0.61 \pm 0.02 \mathrm{aB}$ \\
100 & $2.2 \pm 0.1 \mathrm{aA}$ & $5.0 \pm 0.5 \mathrm{bB}$ & $0.43 \pm 0.01 \mathrm{cA}$ & $0.79 \pm 0.13 \mathrm{bB}$ \\
500 & $4.1 \pm 0.2 \mathrm{bA}$ & $3.7 \pm 0.3 \mathrm{aA}$ & $1.11 \pm 0.03 \mathrm{dA}$ & $1.07 \pm 0.13 \mathrm{cA}$ \\
1000 & $6.5 \pm 0.2 \mathrm{cB}$ & $4.6 \pm 0.5 \mathrm{bA}$ & $1.56 \pm 0.06 \mathrm{eB}$ & $1.12 \pm 0.16 \mathrm{cA}$ \\
\hline
\end{tabular}

Different lower case letters for different tocopherol concentrations in each sample denote significant differences $(p<0.05)$. Different capital letters for different antioxidants in each lipid substrate denote significant differences $(p<0.05)$. 
Table 3 Antioxidant activity (F/ORR) of $\alpha$ - and $\delta$-tocopherol on the inhibition of hydroperoxides.

\begin{tabular}{lcccc}
\hline Toc $(\mathbf{m g} / \mathbf{k g})$ & HL- $\alpha$ & HL- $\boldsymbol{~}$ & HO- $\boldsymbol{\alpha}$ & HO- $\boldsymbol{\delta}$ \\
\hline 10 & 18 & 8 & 26 & 9 \\
50 & 93 & 21 & 78 & 54 \\
100 & $\mathbf{1 1 5}$ & 36 & $\mathbf{9 7}$ & 63 \\
500 & 114 & 111 & 61 & 102 \\
1000 & 83 & $\mathbf{1 5 7}$ & 49 & $\mathbf{1 3 3}$ \\
\hline
\end{tabular}



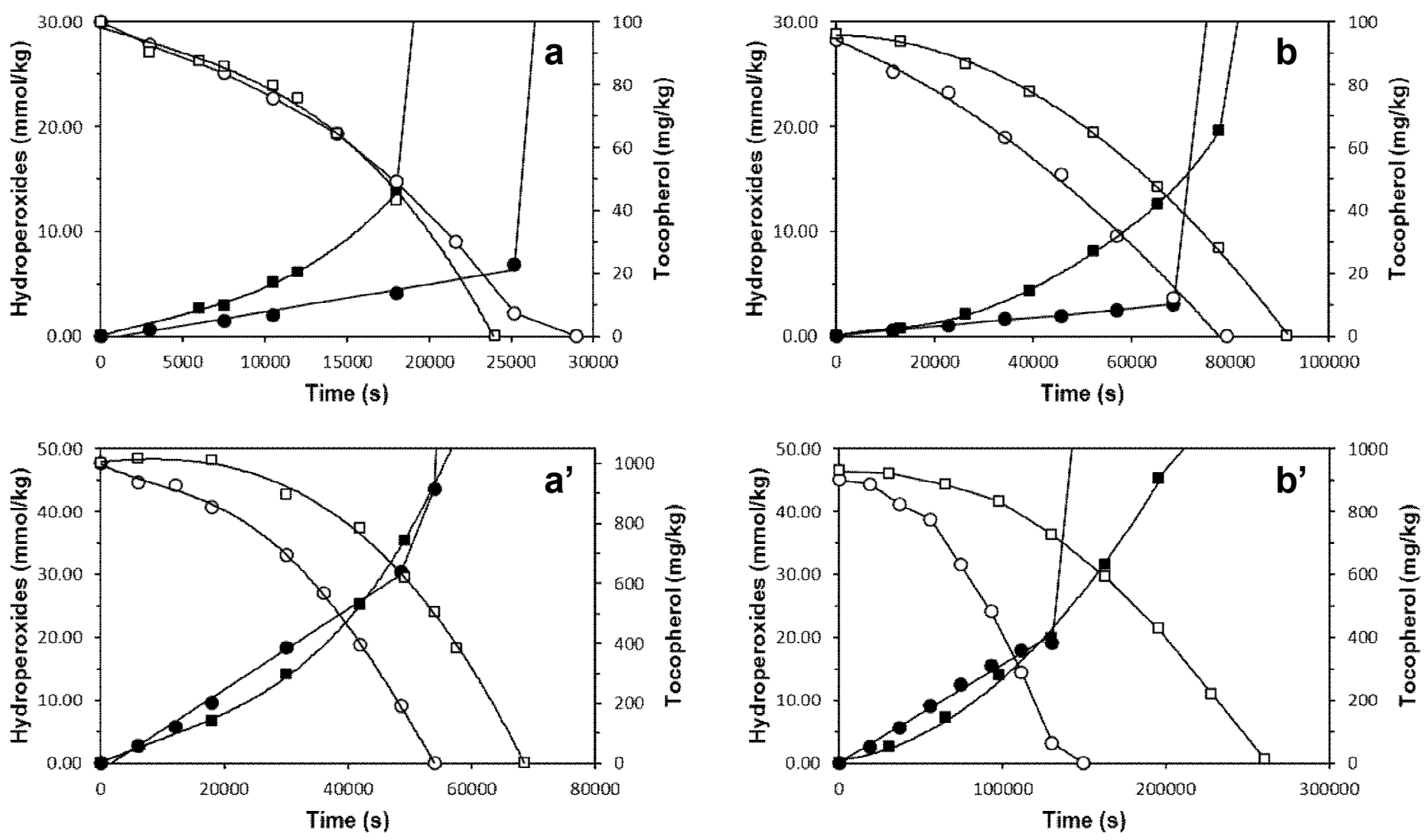

Figure 1 
$\times 10 \mathrm{mg} / \mathrm{kg} \diamond 50 \mathrm{mg} / \mathrm{kg} \triangle 100 \mathrm{mg} / \mathrm{kg} \square 500 \mathrm{mg} / \mathrm{kg} \bigcirc 1000 \mathrm{mg} / \mathrm{kg}$
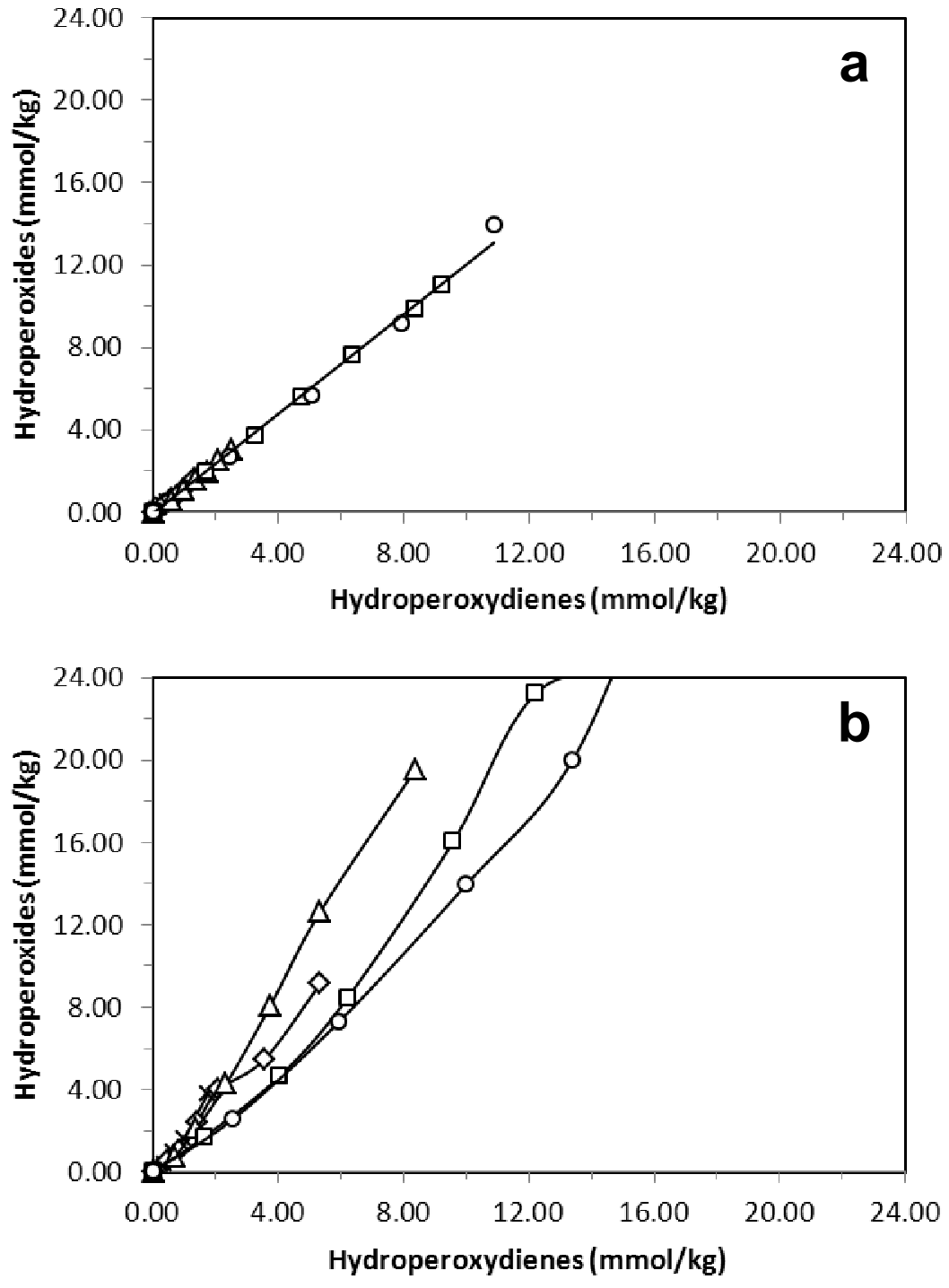

Figure 2 

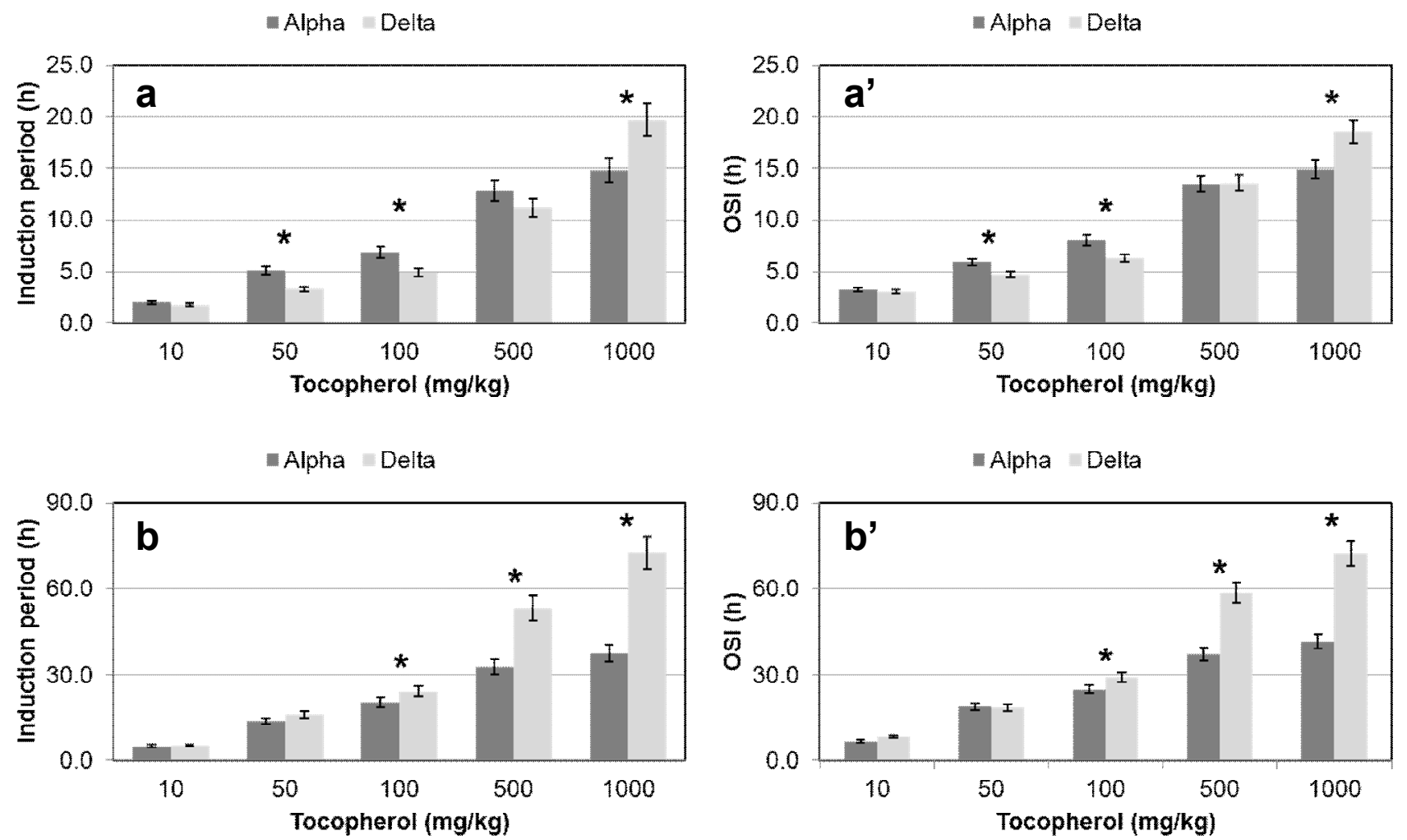

Figure 3 

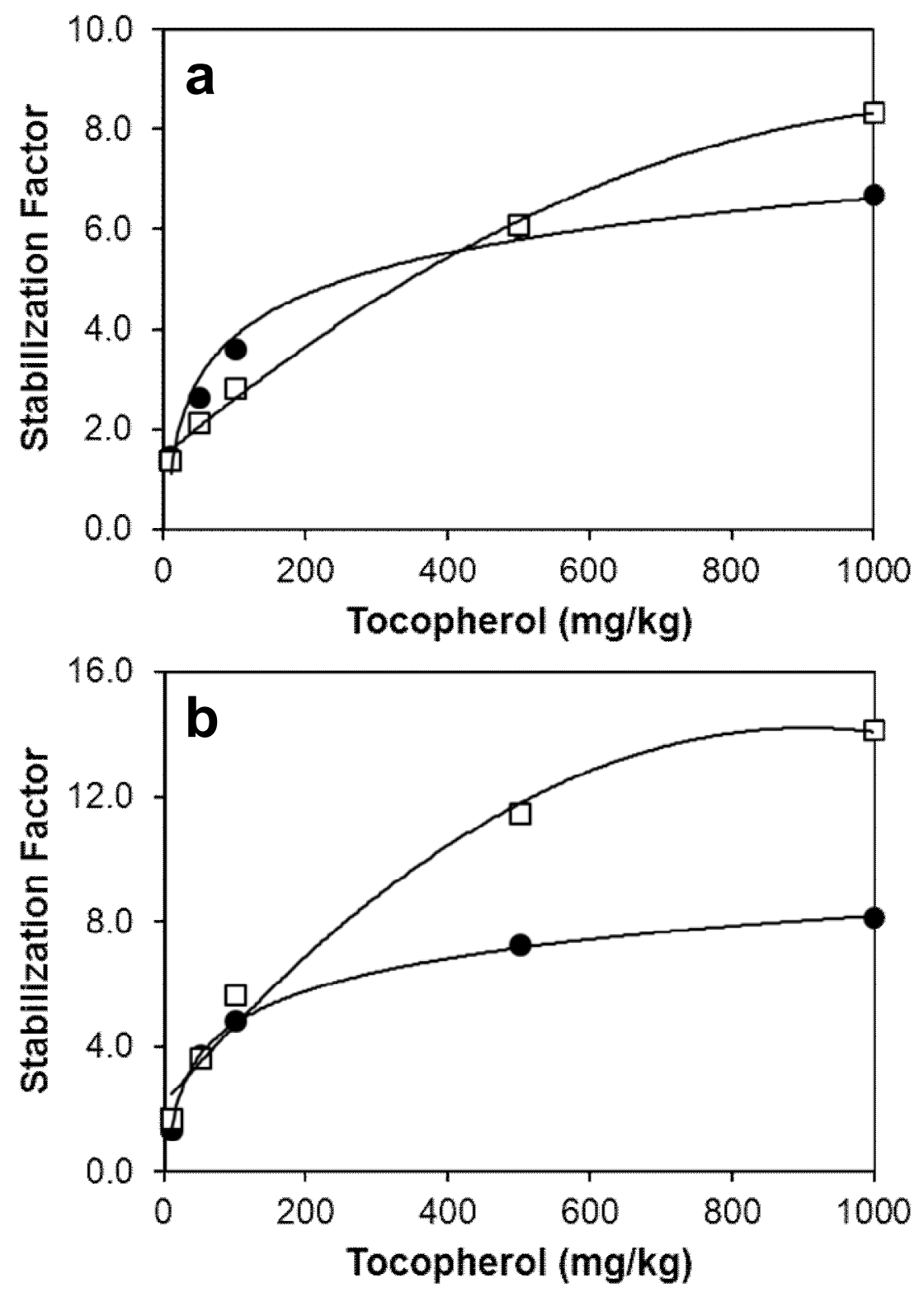

Figure 4 

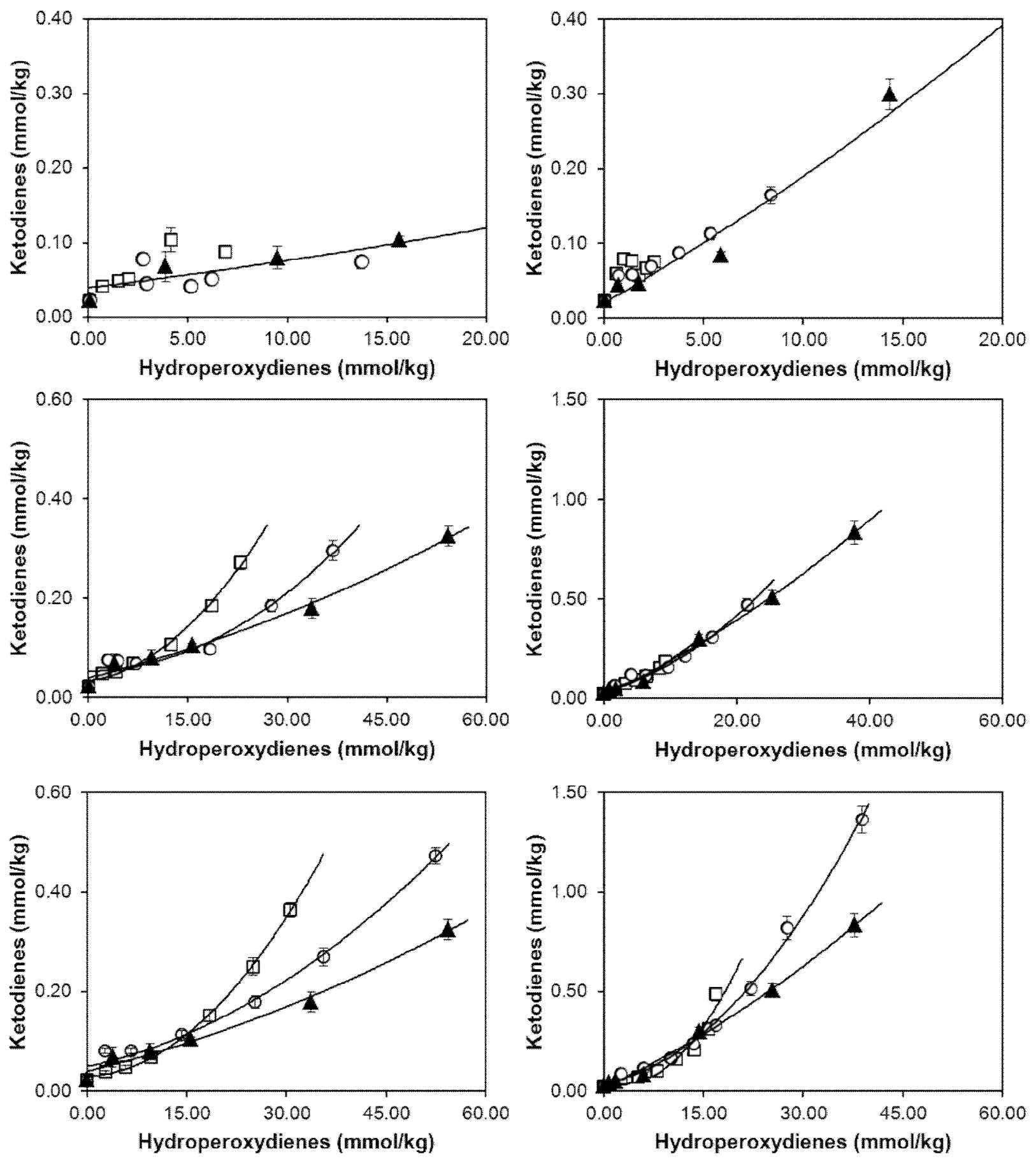

Figure 5 